\title{
Correction of Genu Recurvatum Deformity Using a Hexapod Frame: A Case Series and Review of the Literature
}

\author{
Liam Johnson $^{1}$, James McCammon², Anthony Cooper ${ }^{3}$
}

\begin{abstract}
Aim and objective: Genu recurvatum is a rare deformity for which minimal literature exists. Non-operative management typically gives unsatisfactory results. This study aims to evaluate the treatment of genu recurvatum with a hexapod frame.

Materials and methods: A single-center retrospective chart review of genu recurvatum cases treated with a hexapod fixator application was performed. Radiographic parameters included the following: leg length discrepancy (LLD), angle of recurvatum, angle of tilt of the tibial plateau, patellar height and anatomic proximal posterior tibial angle (aPPTA). Radiographic and functional results were evaluated.

Results: A total of five patients with six limbs corrected with a hexapod frame were found. Aetiology included post-traumatic (2), postinfectious (1) and idiopathic (3). The mean age at application was $13.36(5.5-18.0)$ years. The total mean time in the fixator was 225 (160-412) days. The LLD decreased from a mean of $35.6 \mathrm{~mm}(0.7-50)$ preoperatively to a mean of $14.8(1.0-39.3)$ postoperatively. The average patellar height remained similar $0.97(0.69-1.2)-0.97(0.51-1.6)$. The angle of the tilt of the tibial plateau improved from a preoperative mean of $66^{\circ}$ $\left(58.5-73.5^{\circ}\right)-92.5^{\circ}\left(80-98.5^{\circ}\right)$. The angle of recurvatum improved from a preoperative mean of $26.4^{\circ}\left(18.5-31^{\circ}\right)-5.0^{\circ}\left(0-9^{\circ}\right)$. The aPTTA improved from $\left(102-118^{\circ}\right)-85.5^{\circ}\left(77-96^{\circ}\right)$.

Conclusion: Osteotomy distal to the tibial tuberosity and deformity correction using a hexapod frame allows for multiplanar correction. Throughout treatment, soft tissue management with physical therapy remained key to prevent knee contracture.

Clinical significance: A hexapod frame is a safe and accurate technique that allows correction of genu recurvatum along with concomitant deformities with low risk of complications.

Keywords: Genu recurvatum, Ilizarov, Osteotomy, Taylor spatial frame.

Strategies in Trauma and Limb Reconstruction (2021): 10.5005/jp-journals-10080-1528
\end{abstract}

\section{INTRODUCTION}

Genu recurvatum is excessive hyperextension of the knee and is an uncommon deformity. The specific amount of knee hyperextension varies between authors and is defined between $0^{\circ}$ and $15^{\circ} .^{1-3}$ The clinical sequelae of the deformity may include knee pain, fatigue, weakness, leg length discrepancy, instability, loss of flexion and cosmetic issues. ${ }^{1,2}$ Aetiology varies from idiopathic, trauma, infection and metabolic disorders. Genetic predisposition may be causative and is currently under investigation. ${ }^{4}$ Genu recurvatum can lead to secondary changes in osseous, ligamentous or capsular integrity or a combination of these, further exacerbating the deformity.

In the paediatric population, non-operative management can be compensatory but relies on long-term dependence on bracing with persistent deformity. Surgical techniques consist of proximal tibial osteotomy for an acute correction or more gradual correction with the use of an external fixator frame such as an Ilizarov or hexapod frame. Osteotomies can be divided into opening wedge above the tibial tuberosity, opening wedge below the tibial tuberosity, opening wedge through the tibial tuberosity osteotomy and closing wedge osteotomy. ${ }^{5,6}$ The success of tibial osteotomy depends upon whether the anatomic origin of the genu recurvatum is osseous or soft tissue, as a bony correction does not address the soft tissue component. The osteotomy site, in relation to the patella tendon insertion, will change the soft tissue tension of the extensor mechanism and subsequent patellar height with potential impact on the clinical outcome. ${ }^{5}$
'Division of Surgery and Perioperative Services, Children's Health Queensland Hospital and Health Service, Brisbane, Queensland, Australia

${ }^{2}$ Department of Orthopaedic Surgery, The Children's Hospital of Winnipeg, Winnipeg, Manitoba, Canada; Department of Orthopaedic Surgery, University of Manitoba, Winnipeg, Manitoba, Canada

${ }^{3}$ Department of Orthopaedic Surgery, British Columbia Children's Hospital, Vancouver, British Columbia, Canada; Department of Orthopaedics, Faculty of Medicine, University of British Columbia, Vancouver, British Columbia, Canada

Corresponding Author: Anthony Cooper, Department of Orthopaedic Surgery, British Columbia Children's Hospital, Vancouver, British Columbia, Canada, Phone: +604-875-2000; ext. 6008, e-mail: ExternalFixators@cw.bc.ca

How to cite this article: Johnson L, McCammon J, Cooper A. Correction of Genu Recurvatum Deformity Using a Hexapod Frame: A Case Series and Review of the Literature. Strategies Trauma Limb Reconstr 2021;16(2):116-119.

Source of support: Nil

Conflict of interest: None

Advantages of a hexapod frame correction include a more gradual, multiplanar correction that allows for fine-tuning while respecting the patellar position and maintaining weight bearing status and range of motion (ROM). Another perceived benefit in the growing, skeletally immature paediatric population may be

() Jaypee Brothers Medical Publishers. 2021 Open Access This article is distributed under the terms of the Creative Commons Attribution-Non Commercial-share alike license (https://creativecommons.org/licenses/by-nc-sa/4.0/) which permits unrestricted distribution, and non-commercial reproduction in any medium, provided you give appropriate credit to the original author(s) and the source, provide a link to the Creative Commons license, and indicate if changes were made. If you remix, transform, or build upon the material, you must distribute your contributions under the same license as original. The Creative Commons Public Domain Dedication waiver (http://creativecommons.org/publicdomain/zero/1.0/) applies to the data made available in this article, unless otherwise stated. 
that gradual callotasis maintains the correct soft tissue tension in the extensor mechanism. Previous studies have reported the use of the llizarov frame to treat this deformity in as many as 10 patients with good long-term outcomes. ${ }^{7,8}$ To the best of our knowledge, no previous studies have reported outcomes of genu recurvatum treated with hexapod frames. The aim of this retrospective study was to investigate the success of the correction of genu recurvatum deformity using a hexapod frame. By analyzing the cohort in more detail, we may be able to determine patient and surgical factors that contribute to more successful surgery.

\section{Materials and Methods}

This study involved research involving human participants. All procedures performed in this study were in accordance with the applicable ethical standards. Study approval was obtained from the University of British Columbia Children's and Women's Research Ethics Board (UBC - C\&W REB) prior to the initiation of study activities.

Patient notes, both in paper and electronic form, were accessed for data collection including demographics, operation parameters and outcome measures. All data were deidentified. Inclusion criteria included any patient who underwent genu recurvatum deformity correction with a hexapod fixator in the last 5 years by a single surgeon. This case series has been reported in line with the preferred reporting of caseseries in surgery (PROCESS) Guideline. ${ }^{9}$

\section{Radiographic Analysis}

Full-length standing films were taken in both anteroposterior (AP) and lateral planes before and after treatment. A lateral full-length leg radiograph with the knee in maximum hyperextension was also obtained.

Deformity defined by radiographic parameters included the following: leg length discrepancy, angle of recurvatum, angle of tilt of the tibial plateau, patellar height (according to the Blackburne and Peel method) and the anatomic proximal posterior tibial angle (aPPTA). The aPPTA is defined as the sagittal plane middiaphyseal line that intersects the proximal tibial joint line at an average anatomic axis: joint edge ratio (AJER) of one-fifth the joint line width. The average value of the aPPTA is $81 \pm 44^{\circ}$. $^{10}$ Functional results were evaluated using the Lecuire's anatomic and functional score (Table 1).

Table 1: Lecuire et al. scoring system (Reproduced with permission from: Revue de chirgurige orthopedique et reparatrice de l'appareil moteur)

\begin{tabular}{lc}
\hline Anatomic (radiographic) results & Points \\
\hline Angle of recurvatum (RG) & \\
$0-3^{\circ}$ & 40 \\
$4-6^{\circ}$ & 30 \\
$7-9^{\circ}$ & 20 \\
$10-12^{\circ}$ & 10 \\
$>12^{\circ}$ & 0 \\
Angle of tilt of the tibial plateau & \\
$92-100^{\circ}$ & 30 \\
$88-91^{\circ}$ or $101-104^{\circ}$ & 20 \\
$84-87^{\circ}$ or $105-108^{\circ}$ & 10 \\
$<84^{\circ}$ or $>108^{\circ}$ & 0 \\
Patellar height (A:B ratio) & \\
$0.66-0.94$ & 30
\end{tabular}

$0.51-0.65$ or $0.95-1.09$

$0.36-0.50$ or $1.10-1.24$

$<0.36$ or $>1.24$

Total anatomic (radiographic score)

Excellent

$90-100$

Good

$70-80$

Fair

$40-60$

Poor

Functional results

Pain

None

Slight

10

Mild

5

Severe

Instability

None

15

Mild or slight

5

Severe

ROM

Full

Decreased 1-20

10

Decreased $>20^{\circ}$

0

Weakness

None

15

Slight

10

Mild

5

Severe

Sports activity

Yes

10

No

Patient evaluation of result

Excellent

Good

10

Fair

5

Poor

Total functional score

Excellent

$85-100$

Good

60-80

Fair

40-55

Poor

$<40$

Total combined score

Excellent

175-200

Good

130-170

Fair

$80-125$

Poor

$<80$

\section{Operative Technique}

The patient was positioned supine on the table under general anaesthetic. Fibular osteotomy was performed approximately 10 centimetres $(\mathrm{cm})$ distal to the knee joint, with care to identify and protect the superficial peroneal nerve. The fibula was transfixed to the tibia distal to the fibula osteotomy with a large fragment cortical screw to avoid proximal migration of the distal fibula. An appropriately sized partial ring was placed proximally with the opening posteriorly to allow 
Management of Genu Recurvatum

Table 2: Clinical details and data of case series treated with hexapod

\begin{tabular}{|c|c|c|c|c|c|c|c|c|c|c|}
\hline Case & Age & Diagnosis & $\begin{array}{c}\text { Treatment } \\
\text { duration } \\
\text { (days) }\end{array}$ & $\begin{array}{c}\text { Angle of } \\
\text { recurvatum } \\
\text { pre }\end{array}$ & $\begin{array}{c}\text { Angle of } \\
\text { recurvatum } \\
\text { post }\end{array}$ & $\begin{array}{c}\text { Angle tilt } \\
\text { of plateau } \\
\text { pre }\end{array}$ & $\begin{array}{c}\text { Angle tilt } \\
\text { of plateau } \\
\text { post }\end{array}$ & $\begin{array}{c}\text { Patellar height } \\
\text { pre }\end{array}$ & $\begin{array}{c}\text { Patellar } \\
\text { height post }\end{array}$ & $\begin{array}{l}\text { Anatomicl } \\
\text { functional } \\
\text { score }\end{array}$ \\
\hline 1 & 11.3 & Post-infectious & 177 & 18.5 & 0 & 72.5 & 80 & 1.04 & 0.72 & Good (70/75) \\
\hline $2 \mathrm{R}$ & 15.7 & Idiopathic & 196 & 28.5 & 5.5 & 64 & 96 & 0.69 & 0.94 & Good (90/80) \\
\hline $2 \mathrm{~L}$ & & & & 26.5 & 9 & 62.5 & 88 & 1.045 & 0.87 & Good (70/80) \\
\hline 3 & 14.1 & Post-traumatic & 212 & 31 & 5.5 & 73.5 & 94.5 & 1.215 & 1.2 & Good (70/90) \\
\hline 4 & 5.5 & Post-traumatic & 412 & 29 & 5 & 58.5 & 98.5 & Not measurable & 1.6 & Fair (60/45) \\
\hline 5 & 18 & Idiopathic & 160 & 25 & 5 & 65 & 98 & 0.86 & 0.51 & Good (80/90) \\
\hline
\end{tabular}

knee flexion. A second full ring was placed distally. Placement of the first ring was directed by a guide wire from lateral to medial, parallel and distal to the joint line by approximately 16 millimetres $(\mathrm{mm})$. Rings were secured with tensioned fine wires and $6 \mathrm{~mm}$ hydroxyapatitecoated pins using intermittent drilling. Struts were secured between the proximal and distal ring. Strut measurements were all recorded at the end of the procedure. Tibial osteotomy was performed at the metaphyseal-diaphyseal junction below the tuberosity using a 3.5-mm drill and osteotomes under fluoroscopic guidance. Leg was cleaned with hydrogen peroxide, and silver-impregnated sponges were placed over all the pin sites. Postoperatively, patients were placed on a pain protocol including morphine, pregabalin and lidocaine infusion. Seven days after frame application and osteotomy, distraction was started gradually over a period of approximately 8 weeks. Subsequent to this, the frame was left in situ to allow the bony regenerate to consolidate up removal of the frame. Physiotherapy actively treated patients throughout the course of treatment three times per week, with twice daily home exercises on the intervening days to ensure ROM of the knee was maintained (Table 2).

\section{Results}

A total of five patients with six limbs corrected with a hexapod frame were found (Table 2). Aetiology included post-traumatic (2), postinfectious (1) and idiopathic (3). Five frames were the Taylor Spatial Frame (TSF) (Smith and Nephew) and one frame was the Orthex frame (Orthopediatrics). All patients had a leg length discrepancy, and five out of six had concomitant genu valgum (5). The mean age at application was $13.36(5.5-18.0)$ years. The total mean time in the fixator was 225 (160-412) days. The LLD decreased from a mean of $35.6 \mathrm{~mm}(0.7-50)$ preoperatively to a mean of $14.8 \mathrm{~mm}(1.0-39.3)$ postoperatively. Subsequent staged procedures are planned for two patients to address remaining LLD. The average patellar height remained similar $0.97(0.69-1.2)$ to $0.97(0.51-1.6)$. The angle of the tilt of the tibial plateau improved from a preoperative mean of $66^{\circ}$ $\left(58.5-73.5^{\circ}\right)$ to $92.5^{\circ}\left(80-98.5^{\circ}\right)$. The angle of recurvatum improved from a preoperative mean of $26.4^{\circ}\left(18.5-31^{\circ}\right)$ to $5.0^{\circ}\left(0-9^{\circ}\right)$. The aPPTA improved from $\left(102-118^{\circ}\right)$ to $85.5^{\circ}\left(77-96^{\circ}\right)$. The average functional outcome score (Lecuire score) was good at last follow-up for each patient (range of last follow-up, 2 months to 2 years). One patient reported a fair outcome following the surgery; however, this patient had a pre-existing foot drop from compartment syndrome from intraosseous resuscitation as an infant. There were no new cases of peroneal nerve palsy.

\section{Discussion}

Our results suggest that gradual correction of proximal tibial recurvatum using a hexapod fixator provided good to excellent clinical and radiographic outcomes. Our study focussed on patients with proximal tibial recurvatum as the primary cause of the clinical deformity. Hence, correction of the bony deformity predictably yielded a good outcome for our patients. Although we were able to identify specific insults to the proximal tibial physis in most cases (e.g., trauma/infection), there were some patients in whom no cause was identified. As mentioned previously, genetic predisposition to genu recurvatum is under investigation.

There have been relatively few studies looking at the correction of genu recurvatum using the llizarov frame. Choi et al. ${ }^{8}$ retrospectively analysed in 10 adult patients with genu recurvatum treated using the llizarov method. The angle of recurvatum improved from a mean of $19.6^{\circ}$ to $-3.0^{\circ}$. The angle of tilt of the tibial plateau improved from a mean $76.6^{\circ}$ to $95^{\circ}$ and ipsilateral limb length discrepancy from $27 \mathrm{~mm}$ to less than $5 \mathrm{~mm}$. At a mean of 4.4 year follow-up, three patients had excellent and six had good and fair results based on the Lecuire criteria.

Manohar et al. ${ }^{7}$ analysed treatment of nine patients with genu recurvatum treated with the the llizarov method. Aetiology in the majority was traumatic or idiopathic. The mean age was 14.3 years comprising six males and three females. The angle of recurvatum improved from a mean of $28^{\circ}$ to $7^{\circ}$. The angle of tilt of the tibial plateau improved from $69^{\circ}$ to $93^{\circ}$. At a mean follow-up of 4.4 years, the overall results were good to excellent using the Lecuire criteria.

Segal ${ }^{11}$ treated $75^{\circ}$ bilateral genu recurvatum in a patient with pseudoachondroplasia using an Ilizarov frame over a 9-month period. At 1 year follow-up, the patient was pain free, had no hyperextension of the knee and had flexion of the knees to $100^{\circ}$. She was able to ambulate without walking aids.

The various osteotomy and plating techniques used in the past to treat genu recurvatum have some potential disadvantages. These include incomplete correction, patella baja, knee pain/stiffness and the need to remove metalwork at a later date. ${ }^{5,8}$ There are also complications from bone graft donor site morbidity. ${ }^{12}$ Additionally, there are often other associated deformities such as genu valgum and limb length deformities that may not be amenable to correction with a single osteotomy. Distraction osteogenesis overcomes these complications and can potentially treat multiple deformities with a single procedure, avoiding the need for bone graft. Other advantages include early weight bearing on the limb and a potential shorter hospital stay. The angle between the anterior cortical line of the femur and the anterior cortical line of the tibia was used as a surrogate for the aPPTA for both preoperative planning and to assess correction. It can be difficult to measure the aPPTA if the ring is placed very proximally, as it will obscure the proximal joint line. Maximum hyperextension is needed to calculate this angle. This angle depends on the effort and ability of the radiology 
technicians to obtain maximum hyperextension. Some assistance may be required to help push the patient's knee into maximum hyperextension to ensure accurate effort. It also requires intensive postoperative physiotherapy because if there is an unrecognized knee flexion contracture, there may be unintentional under correction of the deformity.

There are currently no studies in the literature looking at correction of proximal tibia recurvatum using hexapod frames. The results are similar to studies examining the use of an llizarov construct in terms of numbers of patients and outcomes. No complications occurred in this series, in particular, no common peroneal nerve palsies, compartment syndromes and no bone or joint infection. The peroneal nerve is monitored throughout and protected by the gradual correction of the hexapod frame. Infection is a constant possibility with the frame; joint sepsis is limited particularly by careful positioning of pins and wires away from the joint capsule. Finally, there were no significant discrepancies between patellar height radiologically after treatment which is attributed to the osteotomy position as well as a strict regimen of physiotherapy to maintain ROM.

Small patient numbers are a limitation of this study. This is in keeping with the published literature surrounding the operative management of this rare condition. Other limitations of this study include the retrospective nature and variable length of follow-up.

\section{Conclusion}

We conclude from our data that hexapod fixators are a safe and effective surgical technique allowing correction of genu recurvatum and concomitant deformities as well as any associated limb length discrepancy.

\section{Clinical Significance}

Genu recurvatum deformity correction provides a challenging compilation of soft tissue and bony components. Hexapod fixators can address these simultaneously and accurately with low risk of complications.

\section{Acknowledgements}

\section{Manufacturer Name}

Hexapod Frame

- Orthex: Manufacturer: OrthoPediatrics, Warsaw, Indiana
- Taylor Spatial Frame (TSF): Manufacturer: Smith and Nephew London, United Kingdom

Consent: This study presents a retrospective review of data. An informed consent was not required for this retrospective review as per local institutional ethics board.

All procedures performed in studies involving human participants were in accordance with the ethical standards of the institutional and/or national research committee and with the 1964 Helsinki declaration and its later amendments or comparable ethical standards.

\section{References}

1. Brownstein B, Noyes F, Mangine RE, et al. Anatomy and biomechanics. In: Physical therapy of the knee. New York: Churchill Livingstone; 1988. p. 1-30.

2. Loudon JK, Goist HL, Loudon KL. Genu recurvatum syndrome. J Orthop Sport Phys Ther 1998;27(5):361-367. DOI: 10.2519/ jospt.1998.27.5.361.

3. Lecuire $F$, Lerat $J \mathrm{~L}$, Bousquet $\mathrm{G}$, et al. Le genu recurvatum et son traitement par ostéotomie tibiale [The treatment of genu recurvatum (author's transl)]. Rev Chir Orthop Reparatrie Appar Mot 1980;66:95-103. PMID: 6451907.

4. Feczko P, Emans P. Hereditary bilateral genu recurvatum: case report of a family. Knee 2017;24:137-143. DOI: 10.1016/j.knee.2016.09.025.

5. Moroni A, Pezzuto V, Pompili M, et al. Proximal osteotomy of the tibia for the treatment of genu recurvatum in adults. J Bone Jt Surg 1992;74(4):577-586. PMID: 1583053.

6. Nathan ST, Parikh SN. Genu recurvatum after tibial tuberosity fracture. Case Rep Orthop 2013;1-5. DOI: 10.1155/2013/952978.

7. Manohar Babu KV, Fassier F, Rendon JS, et al. Correction of proximal tibial recurvatum using the llizarov technique. J Pediatr Orthop 2012;32(1):35-41. DOI: 10.1097/BPO.0b013e31823b15e2.

8. Choi IH, Chung CY, Cho TJ, et al. Correction of genu recurvatum by the llizarov method. J Bone Jt Surg - Ser B 1999;81(5):769-774. DOI: 10.1302/0301-620x.81b5.9589.

9. Agha RA, Sohrabi C, Mathew G, et al. The PROCESS 2020 Guideline: updating consensus Preferred Reporting Of CasESeries in Surgery (PROCESS) guidelines. Int J Surg 2020;84:231-235. DOI: 10.1016/j. ijsu.2020.11.005.

10. Paley D. Principles of deformity correction. Heidelberg: SpringerVerlag; 2002.

11. Segal O. llizarov treatment for extreme bilateral genu recurvatum in a pseudoachondroplasia patient: a case report. Acta Orthop Belg 2010;76(1):124. PMID: 20306977.

12. Van Raaij TM, De Waal Malefijt J. Anterior opening wedge osteotomy of the proximal tibia for anterior knee pain in idiopathic hyperextension knees. Int Orthop 2006;30(4):248-252. DOI: 10.1007/ s00264-005-0063-x. 